Check for updates

Cite this: RSC AdV., 2018, 8, 4890

\title{
ZIF-8 derived Ag-doped ZnO photocatalyst with enhanced photocatalytic activity
}

\author{
Xiaobing Yang, ${ }^{\text {ab }}$ Liqing Qiu ${ }^{\text {ab }}$ and Xuetao Luo (D) *c
}

Recently, zeolitic imidazolate framework-8 (ZIF-8) has been widely studied and used as a catalyst in various fields, due to its high specific surface area, tunable channels and thermal and chemical stability. In this paper, ZIF-8 was used as a precursor to fabricate a Ag/ZnO photocatalyst, and the influence of $\mathrm{Ag}$ on the photocatalytic activity of $\mathrm{ZnO}$ has been explored. All samples were characterised using XRD, SEM, TEM, and UV-vis diffuse reflectance spectra. The photocatalytic activity of all samples was evaluated by the degradation of a rhodamine B solution under UV light. The results show that ZIF- 8 was completely transformed into $\mathrm{ZnO}$ when it was calcined at $550{ }^{\circ} \mathrm{C}$ for $6 \mathrm{~h}$, and $\mathrm{Ag}$ was well loaded onto $\mathrm{ZnO}$. The photocatalytic efficiency of $\mathrm{ZnO}$ is $92.32 \%$. When $\mathrm{ZnO}$ was doped with $\mathrm{Ag}$, its photocatalytic efficiency was highly improved (99.64\%). Furthermore, Ag/ZnO exhibited high photocatalytic stability. After five repeated cycles, the photocatalytic activity of $\mathrm{Ag} / \mathrm{ZnO}$ was highly retained at $97.48 \%$.

Received 15th December 2017 Accepted 18th January 2018

DOI: 10.1039/c7ra13351k

rsc.li/rsc-advances

\section{Introduction}

To date, photocatalytic degradation has been one effective way of treating various water pollutants, and semiconductor photocatalysts have been widely investigated. Over several decades of studies, many semiconductor photocatalysts have been found, such as $\mathrm{TiO}_{2},{ }^{1,2} \mathrm{SnO}_{2},{ }^{3} \mathrm{ZrO}_{2},{ }^{4}$ and $\mathrm{ZnO} .{ }^{5}$ They possess a high degradation capacity toward toxic and recalcitrant chemical species through relatively simple and low-cost procedures. Among these semiconductor photocatalysts, $\mathrm{ZnO}$, with a band gap of $3.2 \mathrm{eV}$, has been widely investigated, due to its powerful oxidation capability, non-toxicity and chemical stability. ${ }^{6}$ It can degrade organic dyes into non-toxic substances.

In order to expand the usage of $\mathrm{ZnO}$, researchers have made efforts to improve its catalytic efficiency. One of the main problems affecting the deactivation of $\mathrm{ZnO}$ is the high tendency of the hole-electron pair to recombine, so it is a challenge to ensure the effective separation of the electron-hole pairs of ZnO. After a few decades of research, researchers have found that doping with some metals, such as manganese, cobalt, silver, and so on, is a good strategy to overcome this problem. For instance, Ullah et al. used wet-chemical techniques to prepare the Mn-doped $\mathrm{ZnO}$, and found that the photodegradation efficiency of Mn-doped $\mathrm{ZnO}$ was significantly

${ }^{a}$ College of Ecology and Resource Engineering, Wuyi University, Fujian Wuyishan, 354300, China

${ }^{b}$ Fujian Provincial Key Laboratory of Eco-Industrial Green Technology, Wuyi University, Fujian Wuyishan, 354300, China

'Fujian Key Laboratory of Advanced Materials, College of Materials, Xiamen University, Xiamen 361005, China. E-mail: xuetao@xmu.edu.cn higher than that of the undoped $\mathrm{ZnO}^{7} \mathrm{Xu}$ et al. adopted a hydrothermal method to prepare ZnO powders with different $\mathrm{Co}^{2+}$ doping concentrations $(0,0.5,1,3$, and $5 \mathrm{~mol} \%) .{ }^{8}$ When the doping concentration was $3 \mathrm{~mol} \%$, the degradation ratio of MO reached $78 \%$ after reaction for $240 \mathrm{~min}$. Divband et al. employed photo reduction, chemical reduction and polyacrylamide-gel methods to obtain $\mathrm{Ag} / \mathrm{ZnO}$ photocatalysts with different $\mathrm{Ag}$ loadings. ${ }^{9}$ They found that the metallic $\mathrm{Ag}$ in the $\mathrm{Ag} / \mathrm{ZnO}$ photocatalysts facilitated the trapping of photogenerated electrons and improved the photocatalytic activity of the $\mathrm{Ag} / \mathrm{ZnO}$ photocatalysts. $\mathrm{Ag} / \mathrm{ZnO}$ prepared by the polyacrylamide gel method exhibited the best photocatalytic performance, in comparison with that prepared by the chemical reduction and photo reduction methods.

As a kind of MOF, ZIF-8 has a high specific surface area and tunable channels, as well as thermal and chemical stability. ${ }^{10-12}$ Due to its excellent properties, it has been widely used in various catalytic fields such as CO oxidation, ${ }^{13}$ Friedel-Crafts acylation, ${ }^{14}$ and cyclohexene hydrogenation. ${ }^{15}$ ZIF-8 is constructed from Zn(II) and 2-methylimidazole ligands. So, ZIF-8 is expected to be used as a $\mathrm{Zn}$ source to prepare $\mathrm{ZnO}$. In this paper, we adopted a facile method that combines an adsorption method with heat treatment to prepare Ag doped $\mathrm{ZnO}$ by using ZIF-8 as a precursor. We explored the influence of Ag on the properties of the ZnO derived from ZIF-8. The photocatalytic activity of all samples was evaluated by the degradation of rhodamine B in aqueous solution under UV light. After careful studying, Ag/ZnO shows higher photocatalytic activity than the pure $\mathrm{ZnO}$ derived from ZIF-8. We expect that $\mathrm{Ag} / \mathrm{ZnO}$ can be used as a photocatalyst for the degradation of some organic dyes coming from contaminants. 


\section{Experimental}

\subsection{Chemicals}

Zinc nitrate hexahydrate $\left[\mathrm{Zn}\left(\mathrm{NO}_{3}\right)_{2} \cdot 6 \mathrm{H}_{2} \mathrm{O}, \geq 98 \%\right]$, methanol ( $\geq 99 \%)$, ethyl alcohol ( $\geq 99 \%)$, rhodamine B (RhB) and silver nitrate $\left(\mathrm{AgNO}_{3}, \geq 99.8 \%\right)$ were purchased from Sinopharm Chemical Reagent Co. Ltd. 2-Methylimidazole (Hmim, $\geq 99 \%$ ) was obtained from Chengdu Kelong Chemical Reagent Company. All chemicals were used directly without any further purification. Distilled water was used throughout the experiments.

\subsection{Fabrication of the Ag doped $\mathrm{ZnO}$ photocatalyst}

The Ag doped ZnO photocatalyst was derived from ZIF-8. The procedures include the preparation of ZIF-8 and the fabrication of the $\mathrm{Ag}$ doped $\mathrm{ZnO}$ photocatalyst.

ZIF-8 was prepared using our previous research method. Firstly, $10 \mathrm{mmol}$ of $\mathrm{Zn}\left(\mathrm{NO}_{3}\right)_{2} \cdot 6 \mathrm{H}_{2} \mathrm{O}$ was dissolved in $100 \mathrm{~mL}$ of methanol to form the A solution, and $40 \mathrm{mmol}$ of 2-methylimidazole was added into $100 \mathrm{~mL}$ of methanol to form the B solution. Then, the A solution was slowly poured into the B solution with continuous stirring. After five minutes of stirring, the mixture was kept standing at room temperature for $24 \mathrm{~h}$. The mixture was centrifuged and dried at $70{ }^{\circ} \mathrm{C}$ to obtain ZIF-8.

$\mathrm{Ag}$ doped $\mathrm{ZnO}(\mathrm{Ag} / \mathrm{ZnO})$ was prepared from ZIF-8 by the following method: firstly, $0.5 \mathrm{mM} \mathrm{AgNO}{ }_{3}$ was dissolved in $20 \mathrm{~mL}$ of ethanol containing $2 \mathrm{~mL}$ of distilled water to form the $\mathrm{AgNO}_{3}$ solution. Then, $0.5 \mathrm{~g}$ of ZIF-8 was dispersed in the $\mathrm{AgNO}_{3}$ solution with vigorous stirring for $60 \mathrm{~min}$. After that, the $\mathrm{AgNO}_{3}$ treated ZIF-8 was washed with absolute ethanol to remove the residual $\mathrm{Ag}^{+}$adsorbed on the surface of the ZIF-8 nanoparticles, and dried at $50{ }^{\circ} \mathrm{C}$. Finally, the $\mathrm{Ag}$ doped ZIF-8 was calcined at $550{ }^{\circ} \mathrm{C}$ for $6 \mathrm{~h}$, to remove the organic agents and obtain the $\mathrm{Ag} /$ $\mathrm{ZnO}$. The synthetic procedure for the $\mathrm{Ag} / \mathrm{ZnO}$ photocatalyst is shown in Fig. 1.

\subsection{Characterization of the products}

X-ray powder diffraction (XRD) data were recorded on a BrukerAxsD8 diffractometer using $\mathrm{Cu}-\mathrm{K} \alpha$ radiation in the angular range ( $2 \theta$ ) from 5 to $60^{\circ}$, operated at $40 \mathrm{kV}$ and $40 \mathrm{~mA}$. Scanning electron microscope (SEM) images were directly observed using a Hitachi SU70 scanning microscope at an accelerating voltage of $5 \mathrm{kV}$. Electron transmission microscopy (TEM) images were obtained using an F30 under an accelerating voltage of $100 \mathrm{kV}$. UV-vis absorption spectra were measured on a UV-101 PC scanning spectrophotometer.

\subsection{Catalytic activity testing}

Rhodamine $\mathrm{B}(\mathrm{RhB})$ was used as a representative dye to test the photocatalytic activity of all the samples under UV light. Firstly, $0.5 \mathrm{~g}$ of the photocatalyst was dispersed in $100 \mathrm{~mL}$ of a nicotine solution $\left(2 \times 10^{-2} \mathrm{~g} \mathrm{~L}^{-1}\right)$, and kept stirring for $30 \mathrm{~min}$ in the dark to reach an adsorption-desorption equilibrium between $\mathrm{RhB}$ and the photocatalyst. Then, a $300 \mathrm{~W}$ lamp immobilized on top of the reactor was turned on (the distance between the lamp and reactor was $10 \mathrm{~cm}$ ). At regular intervals during this photocatalytic process, several milliliters of the suspension solution were withdrawn from the reactor, centrifuged to remove the catalyst, and the $\mathrm{RhB}$ concentration in solution was measured using a UV-vis spectrophotometer.

\section{Results and discussion}

The X-ray diffraction (XRD) patterns of ZIF-8, ZnO and $\mathrm{Ag} / \mathrm{ZnO}$ are shown in Fig. 2. Fig. 2a shows distinct peaks at $7.38^{\circ}$, $10.42^{\circ}, 12.77^{\circ}, 14.75^{\circ}, 16.50^{\circ}, 18.08^{\circ}$ and $19.53^{\circ}$, which are ascribable to the (011), (002), (112), (022), (013), (222) and (123) reflections of ZIF-8, respectively. ${ }^{16}$ When ZIF-8 is calcined at $550{ }^{\circ} \mathrm{C}$ for $6 \mathrm{~h}$, the original peaks corresponding to ZIF-8 are no longer present. However, it exhibits new peaks at $31.76^{\circ}, 34.44^{\circ}$, $36.25^{\circ}, 47.54^{\circ}, 56.58^{\circ}, 62.85^{\circ}, 66.35^{\circ}, 67.93^{\circ}$ and $69.08^{\circ}$ (Fig. $2 b$ ), which are ascribable to the (100), (022), (101), (102), (110), (103), (200), (112) and (201) reflections of ZnO, respectively. ${ }^{17}$ Fig. $2 \mathrm{c}$ shows the XRD pattern of $\mathrm{Ag} / \mathrm{ZnO}$. In addition to the diffraction peaks of $\mathrm{ZnO}$, it shows new peaks at $38.10^{\circ}, 44.29^{\circ}$ and $64.41^{\circ}$, which are ascribable to the (111), (200) and (220) reflections of $\mathrm{Ag}$, respectively.$^{18}$ These results indicate that ZIF-8 is absolutely transformed into $\mathrm{ZnO}$, and that $\mathrm{Ag}$ is well loaded onto the $\mathrm{ZnO}$.

The morphology of all the samples was obtained using a scanning electron microscope, and the results are shown in Fig. 3. Fig. 3a shows the SEM image of ZIF-8, which exhibits a dodecahedral morphology. When ZIF-8 is calcined at $550{ }^{\circ} \mathrm{C}$ for $6 \mathrm{~h}$, the structure is collapsed (Fig. $3 \mathrm{~b}$ ), and the particle size decreases from $300 \mathrm{~nm}$ to $100 \mathrm{~nm}$. When ZIF-8 is treated with
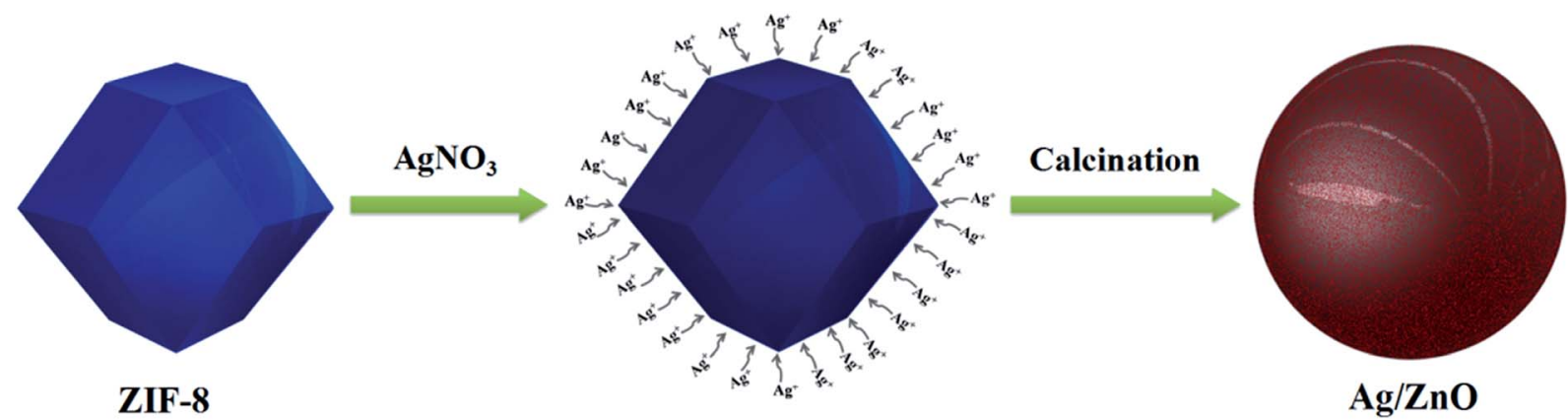

Fig. 1 The synthetic procedure for the $\mathrm{Ag} / \mathrm{ZnO}$ photocatalyst. 


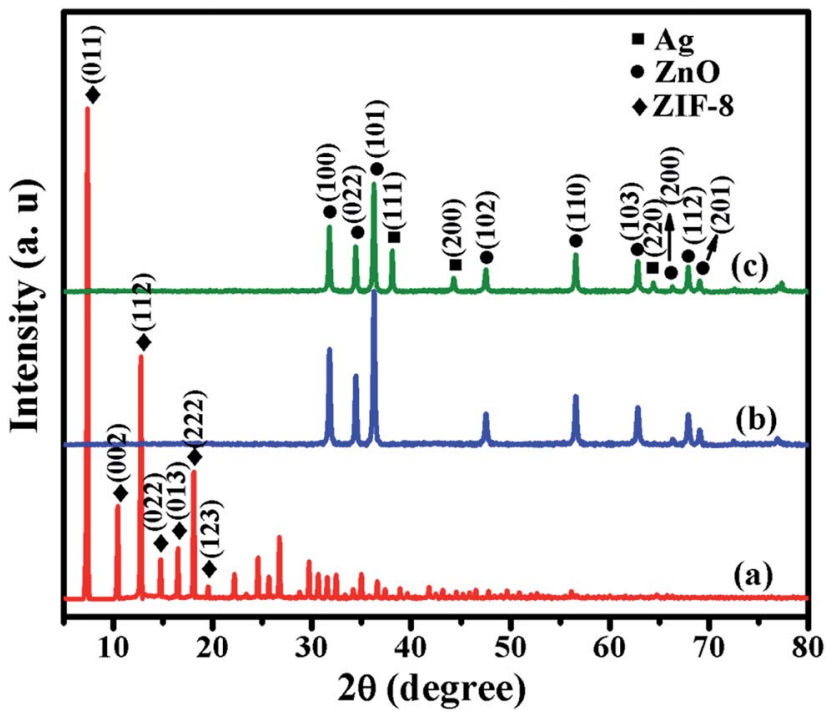

Fig. 2 XRD patterns of (a) ZIF-8, (b) ZnO, and (c) Ag/ZnO.
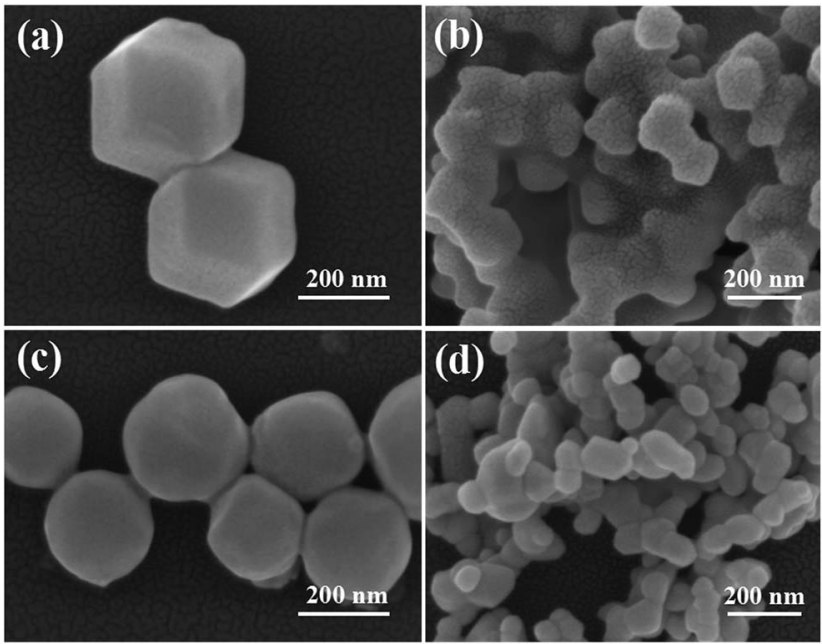

Fig. 3 SEM images of (a) ZIF-8, (b) $\mathrm{ZnO}$, (c) ZIF-8 treated with $\mathrm{AgNO}_{3}$ and (d) $\mathrm{Ag} / \mathrm{ZnO}$.

$\mathrm{AgNO}_{3}$, its edges become rounded (Fig. 3c), which is consistent with Wee's report. ${ }^{16}$ Fig. 3d shows the SEM image of $\mathrm{Ag} / \mathrm{ZnO}$. The particles of ZIF-8, treated with $\mathrm{AgNO}_{3}$, are also collapsed.

Fig. 4 shows the EDS maps of $\mathrm{Ag} / \mathrm{ZnO}$; the EDS images confirm the presence of the elements $\mathrm{Zn}$, O and Ag. Ag is well loaded and dispersed in $\mathrm{ZnO}$ nanoparticles. This indicates that the organic ligands of ZIF- 8 are absolutely removed when it is calcined at $550{ }^{\circ} \mathrm{C}$ for $6 \mathrm{~h}$.

Fig. 5 shows the UV-vis absorption spectra of ZIF-8, $\mathrm{ZnO}$ and $\mathrm{Ag} / \mathrm{ZnO}$. The spectrum of ZIF-8 shows a sharp peak at $252 \mathrm{~nm}$, which is attributed to the excitonic absorption of ZIF-8. When ZIF-8 is calcined at $550{ }^{\circ} \mathrm{C}$ for $6 \mathrm{~h}$, it exhibits a strong absorption peak at $388 \mathrm{~nm}$. This indicates that ZIF-8 is completely transformed into $\mathrm{ZnO}$, which is consistent with the XRD patterns (Fig. 2b). When ZIF-8 is treated with $\mathrm{AgNO}_{3}$ and calcined at $550{ }^{\circ} \mathrm{C}$ for $6 \mathrm{~h}$, its characteristic absorption, corresponding to

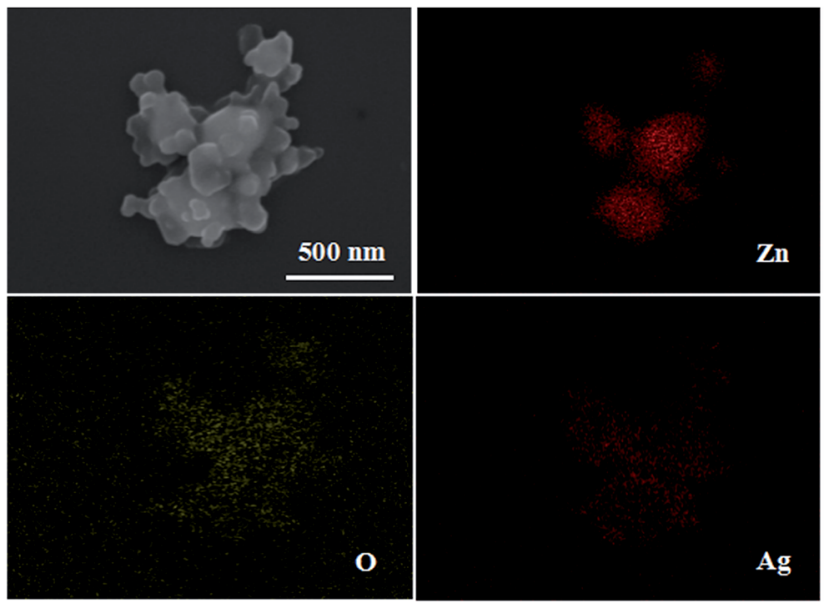

Fig. 4 The EDS maps of $\mathrm{Ag} / \mathrm{ZnO}$.

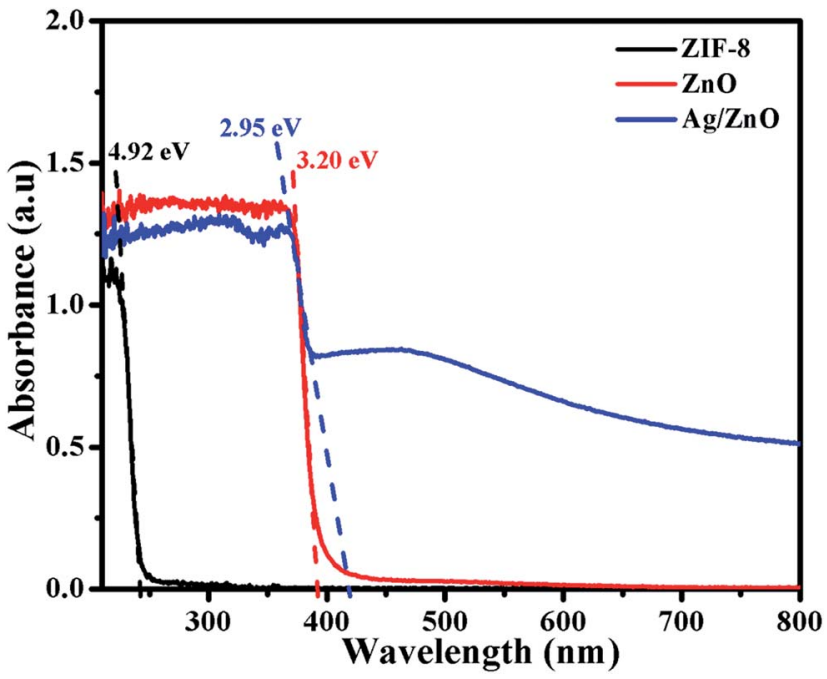

Fig. 5 UV-vis spectra of ZIF-8, ZnO, and Ag/ZnO.

$\mathrm{ZnO}$, exhibits a red shift to $420 \mathrm{~nm}$. Compared to $\mathrm{ZnO}$, the $\mathrm{Ag}$ doped $\mathrm{ZnO}(\mathrm{Ag} / \mathrm{ZnO})$ has a broad absorption in the range of 400-600 nm, which is probably caused by the strong interfacial coupling between the neighbouring ZnO and Ag NPs. ${ }^{17}$ The band gap of the sample can be obtained by the following formula: ${ }^{18}$

$$
A=\left[k\left(h \nu-E_{\mathrm{g}}\right) 1 / 2\right] / h \nu
$$

where $A$ is the absorbance, $k$ is a constant, $h$ is the Planck's constant, $\nu$ is the frequency of light, and $E_{\mathrm{g}}$ is the band gap of the sample. According to the results shown in Fig. 5, the band gaps of ZIF-8 and ZnO are $4.92 \mathrm{eV}$ and $3.20 \mathrm{eV}$, respectively. $\mathrm{Ag} /$ $\mathrm{ZnO}$ has a band gap of $2.95 \mathrm{eV}$, and has a broad band gap in the range of $3.10-2.07 \mathrm{eV}$.

The surface morphologies of $\mathrm{ZIF}-8$ and $\mathrm{Ag} / \mathrm{ZnO}$ were analyzed using low magnification TEM images and HRTEM images. Fig. 6a shows the low magnification TEM image of ZIF8. ZIF-8 displays a dodecahedral morphology. Fig. $6 \mathrm{~b}$ shows the 


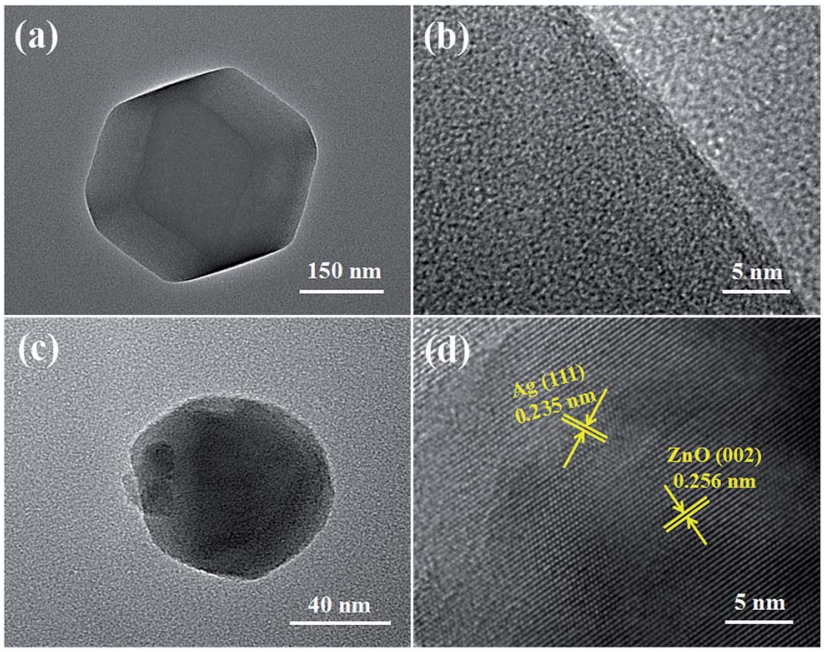

Fig. 6 Low magnification TEM images and HRTEM images of ( $a$ and $b$ ) $\mathrm{ZIF}-8$ and (c and d) $\mathrm{Ag} / \mathrm{ZnO}$.

HRTEM image of ZIF-8. ZIF-8 displays a mesoporous structure, which is in accordance with Pan's report. ${ }^{19}$ Fig. $6 \mathrm{c}$ shows the low magnification TEM image of $\mathrm{Ag} / \mathrm{ZnO}$; the particle size of $\mathrm{Ag} / \mathrm{ZnO}$ is about $100 \mathrm{~nm}$. The high-resolution TEM (HRTEM) (Fig. 6d) of the region shows the characteristic lattice fringes of $0.235 \mathrm{~nm}$ and $0.256 \mathrm{~nm}$, which are assigned to the (111) plane of $\mathrm{Ag}$ and (002) plane of $\mathrm{ZnO}$, respectively. The Ag nanoparticles are well dispersed in $\mathrm{ZnO}$.

The photocatalytic activity of all the photocatalysts was evaluated by the degradation of a RhB solution, and the results are shown in Fig. 7. Fig. 7a shows the photocatalytic degradation of RhB by all the photocatalysts under UV light. In order to eliminate the influence of the adsorption of the photocatalyst, every experiment was carried out in the dark for $30 \mathrm{~min}$. After 30 min of dark reaction, $55.67 \%$ of RhB was adsorbed by ZIF-8, and $17.12 \%$ and $16.43 \%$ of $\mathrm{RhB}$ were adsorbed by $\mathrm{ZnO}$ and $\mathrm{Ag} /$ $\mathrm{ZnO}$, respectively. Then, they were exposed to UV light. After 16 min of irradiation, $12.57 \%$ of RhB was self-degraded. ZIF-8 showed almost no degradation properties, ZnO could degrade
$92.32 \%$ of $\mathrm{RhB}$, and the photocatalytic efficiency of $\mathrm{Ag} / \mathrm{ZnO}$ was 99.64\%. Doping with Ag could highly increase the photocatalytic activity of $\mathrm{ZnO}$. Fig. $7 \mathrm{~b}$ shows the kinetics of all the photocatalysts for the degradation of RhB. The photocatalytic degradation kinetic reaction can be described by $\ln \left(C_{0} / C\right)=k t$ (where $k$ is a pseudo-first-rate kinetic constant and $t$ is the irradiation time). The calculated $k$ value of $\mathrm{ZnO}$ is $0.154 \mathrm{~min}^{-1}$, but the $k$ value of $\mathrm{Ag} / \mathrm{ZnO}$ is $0.241 \mathrm{~min}^{-1}$, which is 1.56 times higher than that of $\mathrm{ZnO} ; \mathrm{Ag} / \mathrm{ZnO}$ shows the highest kinetic constant.

In order to evaluate the photocatalytic stability of the photocatalyst, $\mathrm{Ag} / \mathrm{ZnO}$ was chosen to degrade $\mathrm{RhB}$ for four photocatalytic degradation cycles under UV light. The results are shown in Fig. 8. After four photocatalytic degradation cycles, the photocatalytic efficiency of $\mathrm{Ag} / \mathrm{ZnO}$ reduces by just $2.16 \%$; $\mathrm{Ag} /$ ZnO exhibits high photocatalytic stability. The slight reduction of the photocatalytic efficiency is probably caused by the inevitable loss of the catalyst during the washing process.

Fig. 9 shows the schematic diagram for the photocatalytic degradation of $\mathrm{RhB}$ on $\mathrm{Ag} / \mathrm{ZnO}$. The band gap of $\mathrm{ZnO}$ is $3.20 \mathrm{eV}$. When $\mathrm{ZnO}$ is doped with $\mathrm{Ag}$, it has more defects on its grain boundary. $\mathrm{Ag} / \mathrm{ZnO}$ can be excited by light with a wavelength of less than $400 \mathrm{~nm}$, promoting the valence band electrons up to the conduction band and leaving holes in the valence band as shown in eqn (2). ${ }^{\mathbf{2 0 , 2 1}}$ The holes, produced in the valence band, can adsorb the electrons from $\mathrm{OH}^{-}$or water molecules to form oxidants such as hydroxyl radicals $\left({ }^{\circ} \mathrm{OH}\right)$, as shown in eqn (3). The electrons, left in the conduction band, can combine with the dissolved oxygen to form $\mathrm{O}_{2}{ }^{-}$, as shown in eqn (4). ${ }^{\circ} \mathrm{OH}$ and $\mathrm{O}_{2}{ }^{-}$have strong oxidability and can mineralize RhB molecules, as shown in eqn (5).

$$
\begin{gathered}
\mathrm{Ag} / \mathrm{ZnO}+h \nu \rightarrow \mathrm{Ag} / \mathrm{ZnO}\left(\mathrm{e}^{-}+\mathrm{h}^{+}\right) \\
\mathrm{h}^{+}+\mathrm{OH}^{-} \rightarrow \cdot \mathrm{OH} \\
\mathrm{e}^{-}+\mathrm{O}_{2} \rightarrow \mathrm{O}_{2}^{-} \\
\cdot \mathrm{OH}+\mathrm{O}_{2}^{-}+\mathrm{RhB} \rightarrow \text { degraded products }
\end{gathered}
$$
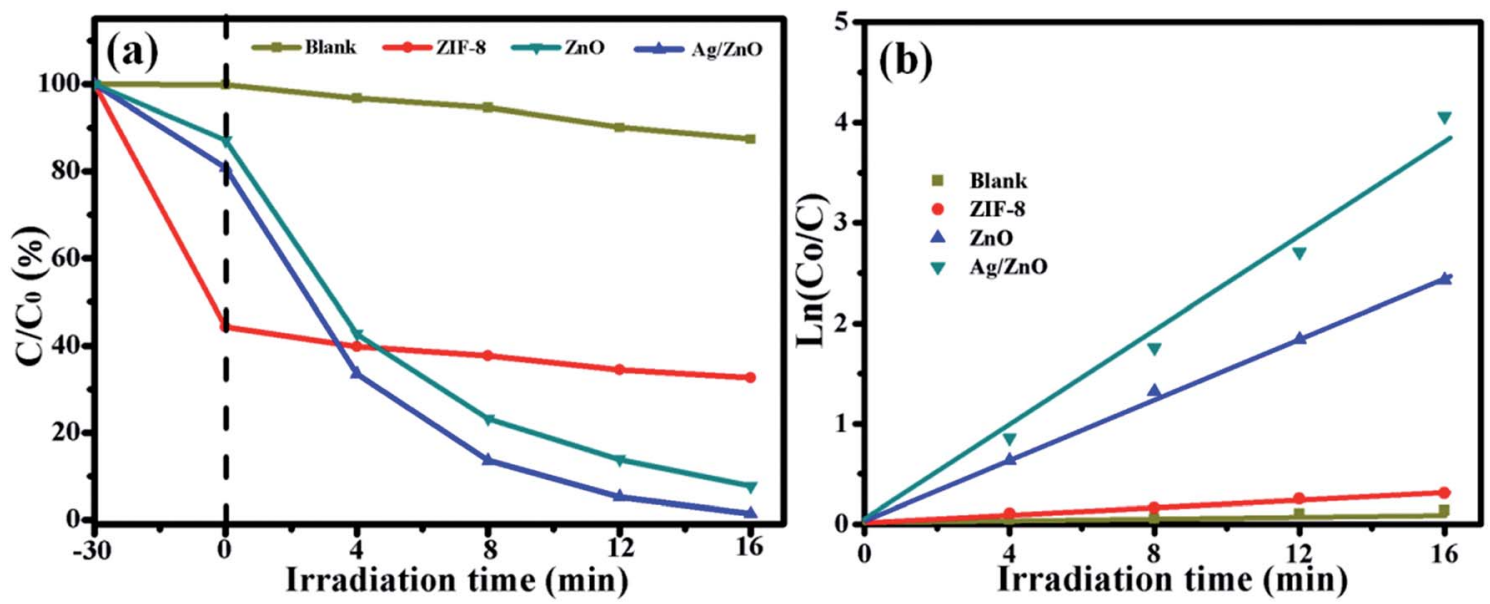

Fig. 7 The photocatalytic degradation (a) and the kinetics (b) of blank, ZIF-8, ZnO, and Ag/ZnO, for the absorption of RhB in the dark and degradation of RhB under UV light irradiation. 


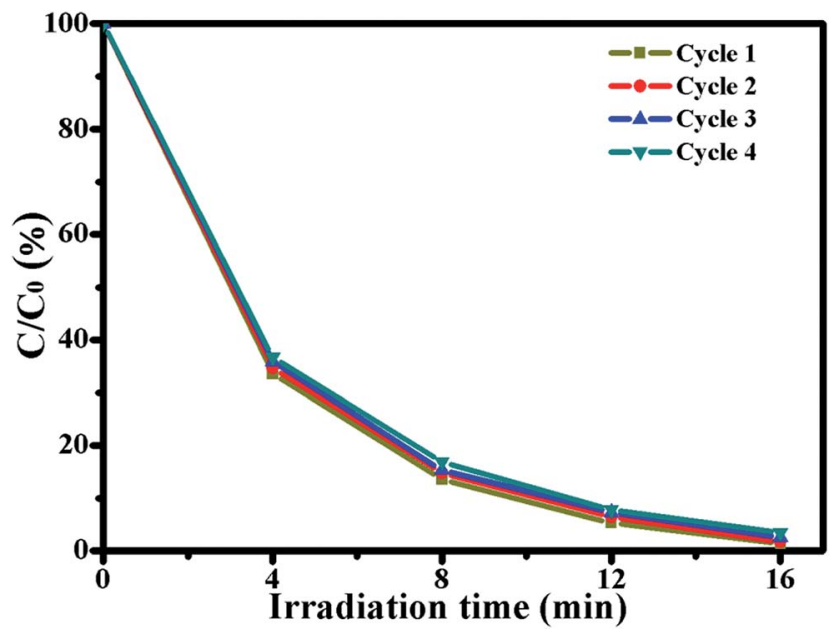

Fig. 8 Four photocatalytic degradation cycles of RhB using Ag/ZnO under UV light.

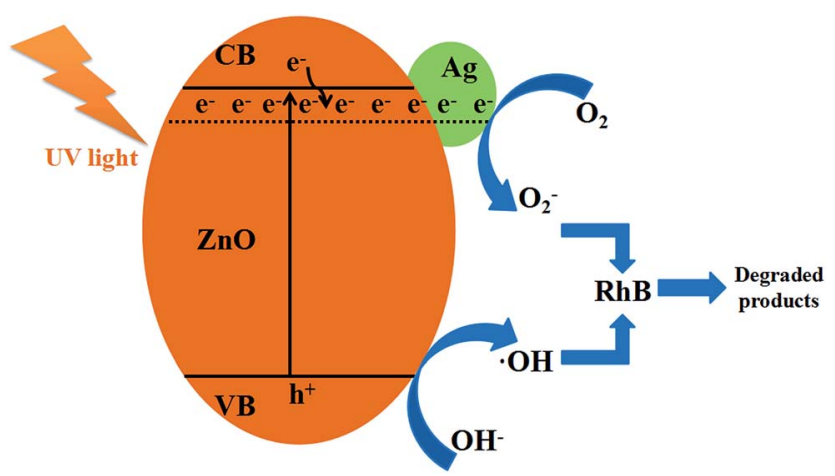

Fig. 9 The schematic illustration of RhB degradation by $\mathrm{Ag} / \mathrm{ZnO}$ under UV light.

\section{Conclusions}

In summary, $\mathrm{Ag}$ doped $\mathrm{ZnO}$, with enhanced photocatalytic activity, was fabricated from a ZIF-8 precursor through an adsorption method and sintering method. Ag was well loaded onto $\mathrm{ZnO}$, which was prepared from ZIF-8. The activity has been successfully improved compared to that of ZIF-8, as the absorption peak of $\mathrm{ZnO}$ was expanded into the red wavelength region, and the photocatalytic efficiency of $\mathrm{ZnO}$ was increased from $92.32 \%$ to $99.64 \%$. Furthermore, $\mathrm{Ag} / \mathrm{ZnO}$ exhibited high photocatalytic stability. After four photocatalytic degradation cycles, the photocatalytic efficiency of $\mathrm{Ag} / \mathrm{ZnO}$ was highly retained at $97.48 \%$. The excellent properties of $\mathrm{Ag} / \mathrm{ZnO}$ make it possible for it to be used for the degradation of organic dyes.

\section{Conflicts of interest}

There are no conflicts to declare.

\section{Acknowledgements}

The authors gratefully acknowledge the financial support of the Fujian Key Laboratory of Advanced Materials, the Scientifi Research Project of Wuyi University (YJ201706), and the Open Project Program of the Provincial Key Laboratory of Eco-Industrial Green Technology (Wuyi University, WYKF2018-8).

\section{References}

1 X. B. Yang, L. Q. Huang, G. Q. Du and X. T. Lu, J. Porous Mater., 2017, 24, 881-886.

2 J. X. Low, B. Cheng and J. G. Yu, Appl. Surf. Sci., 2017, 392, 658-686.

3 K. Maeda and K. Domen, Bull. Chem. Soc. Jpn., 2016, 89, 627648.

4 X. Guo, Chem. Mater., 2004, 16, 3988-3994.

5 X. B. Yang, J. Chen, H. X. Lai, J. P. Hu, M. Fang and X. T. Luo, RSC Adv., 2017, 7, 38519-38525.

6 J. F. Ma, K. Wang, L. Y. Li, T. L. Zhang, Y. Kong and S. Komameni, Ceram. Int., 2015, 41, 2050-2056.

7 R. Ullah and J. Dutta, J. Hazard. Mater., 2008, 156, 194-200. 8 C. Xu, L. X. Cao, G. Su, W. Liu, X. F. Qu and Y. Q. Yu, J. Alloys Compd., 2010, 497, 373-376.

9 B. Divband, M. Khatamian, G. R. K. Eslamian and M. Darbandi, Appl. Surf. Sci., 2013, 284, 80-86.

10 N. L. Torad, M. Hu, S. Ishihara, H. Sukegawa, A. A. Belik, M. Imura, K. Ariga, Y. Sakka and Y. Yamauchi, Small, 2014, 10, 2096-2107.

11 M. Ahmad, E. Ahmed, Z. L. Hong, J. F. Xu, N. R. Khalid, A. Elhissi and W. Ahmed, Appl. Surf. Sci., 2013, 274, 273-281.

12 Y. Q. Chen, J. T. Li, G. H. Yue and X. T. Luo, Nano-Micro Lett., 2017, 9, 32.

13 H. L. Jiang, B. Liu, T. Akita, M. Haruta, H. Sakurai and Q. Xu, J. Am. Chem. Soc., 2009, 131, 11302-11303.

14 L. T. L. Nguyen, K. K. A. Le and N. T. S. Phan, Chin. J. Catal., 2012, 33, 688-696.

15 C. H. Kuo, Y. Tang, L. Y. Chou, B. T. Sneed, C. N. Brodsky, Z. P. Zhao and C. K. Tsung, J. Am. Chem. Soc., 2012, 134, 14345-14348.

16 L. H. Wee, N. Janssens, S. P. Sree, C. Wiktor, E. Gobechiya, R. A. Fischer, C. E. A. Kirschhock and J. A. Martens, Nanoscale, 2014, 6, 2056-2060.

17 S. Ghosh, V. S. Goudar, K. G. Padmalekha, S. V. Bhat, S. S. Indi and H. N. Vasan, RSC Adv., 2012, 2, 930-940.

18 T. J. Whang, M. T. Hsieh and H. H. Chen, Appl. Surf. Sci., 2012, 258, 2796-2801.

19 Y. C. Pan, Y. Y. Liu, G. F. Zeng, L. Zhao and Z. P. Lai, Chem. Commun., 2011, 47, 2071-2073.

20 Y. Q. Chen, J. T. Li, G. H. Yue and X. T. Luo, Nano-Micro Lett., 2017, 9, 32.

21 X. B. Yang, J. Chen, H. X. Lai, J. P. Hu, M. Fang and X. T. Luo, RSC Adv., 2017, 7, 38519-38525. 